\title{
Capital de Marca desde la perspectiva del consumidor
}

\author{
Saavedra Torres, José Luis*
}

\section{Resumen}

El objetivo de este artículo es explorar los conceptos teóricos del Capital de Marca desde la perspectiva del comportamiento del consumidor, como un intento de revisar los basamentos que constituyen su formación. Los planteamientos realizados forman parte de una investigación cualitativa de las propuestas de cuatro autores que formulan modelos para definir las fuentes, métodos para construir y elementos para determinar los beneficios del Capital de Marca, tanto para la organización, como para el consumidor. Se concluye en que el desarrollo teórico del Capital de Marca no ha finalizado aún ya que los modelos aquí analizados se complementan entre sí a la hora de descomponer y analizar sus elementos constitutivos; presentándose la ausencia de una convención ampliamente aceptada sobre la definición del concepto de Capital de Marca.

Palabras clave: Marca, Capital de Marca, Consumidor, Comportamiento del Consumidor, Organización.

\section{Brand Name Markets From the Consumer Perspective}

\section{Abstract}

The objective of this paper is to explore theoretical concepts related to brand name markets from the perspective of the consumer in an attempt to review the foundations that constitute their development. This proposal is part of a qualitative research effort proposed by four authors who formulated models to define sources, methods for their construction, and elements that determine benefits of brand names as capital from the consumer perspective. The conclusion is that brand name capital is still important, even when the models analyzed herein complement each other in the analysis of constituent elements; presenting the absence of a widely accepted convention around the definition of brand name capital.

Key words: Brand name, brand name capital, consumer, consumer behavior, organization.

Recibido: 04-03-17. Aceptado: 04-07-23

Master en Administración (IESA, 1999), Profesor Asociado-Invitado de Mercadeo, FACESLUZ; y Profesor Asociado de Mercadeo, URBE. E-mail: jlsaa@yahoo.com 


\section{Introducción}

La tendencia organizacional de los años 80 de fusión, adquisición y consolidación de empresas y marcas, generaron un caldo de cultivo propicio para el desarrollo de la investigación en el campo de las marcas (Seetharaman et al. 2001). En este escenario, se extendió la idea que las características intangibles asociadas a las marcas son una fuente de riqueza tangible, pues ofrecen una plataforma para la ventaja competitiva y la obtención de ganancias futuras; por lo que las actividades de creación, desarrollo y/o adquisición de marcas ya establecidas en el mercado son consideradas en la actualidad como alternativas serias de inversión (Delgado, 2004).

A partir de la década de los noventa y desde la publicación en 1991 del libro de Aaker "Managing Brand Equity: Capitalizing on the Value of a Brand Name, el concepto de Capital de Marca generó gran interés en el mundo del mercadeo ya que prometió materializar, en conceptos y parámetros financieros, atributos intangibles y difícilmente cuantificables, propios del comportamiento del consumidor (Fernández, 2002).

Las conclusiones de Aaker (1992) sobre la posibilidad de obtener retornos 0 beneficios, tanto para la empresa como para el consumidor, generó el desarrollo de dos orientaciones diferentes. Por un lado la corriente que hace referencia a las manifestaciones económico-financieras de la marca desde un punto de vista empresarial y por otro, la vertiente que analiza la marca en términos del efecto que este elemento tiene en el comportamiento del consumidor (Delgado, 2004).
Ambas orientaciones, han provocado interpretaciones del término Capital de Marca que, aun siendo complementarias, ponen de manifiesto el poco acuerdo que existe sobre su significado (Schultz, 1997; Srivastava y Shocker, 1991; citados por Delgado, 2004). La falta de cohesión en su conceptualización ha generado la inexistencia de instrumentos de medición, pues no hay una definición ampliamente aceptada que haya sido operacionalizada, probada y validada (Delgado, 2004), muy a pesar de los 273 artículos publicados en revistas científicas en los últimos 13 años (Proquest, 2004).

De allí surge el objetivo de este artículo que es explorar los conceptos teóricos del Capital de Marca desde la perspectiva del comportamiento del consumidor, para poder explicar los basamentos que constituyen la formación del concepto.

La justificación para concentrar el análisis en las perspectiva del comportamiento del consumidor, radica en que la literatura referida a la valoración financiera del Capital de Marca reconoce la existencia y la discrecionalidad de este comportamiento, expresado en activos intangibles, como punto de partida para posteriores estudios contables (Woodruff, 1997; Hunt y Morgan, 1995; Slater, 1997; Falkenberg, 1996; Hitt et al, 2001 y Falkenberg, 1996; citados por Delgado, 2004). En este sentido la marca, como activo intangible, posee una naturaleza inmaterial, que necesita ser experimentada y percibida por los consumidores finales para que se construya el Capital de Marca (Delgado, 2004).

El análisis teórico se desarrollará en cuatro fases. Inicialmente se hará una 
revisión de los antecedentes de las dos orientaciones. El segundo punto, es presentar el aporte que la Teoría del Comportamiento al Consumidor plantea sobre el proceso de toma de decisiones de compra y las marcas. El Tercer punto, presentará una revisión de cuatro artículos relacionados con el concepto de Capital de Marca: Farquhar (1989), Aaker (1992), Keller (1993) y Faircloth et al. (2001). Aaker y Keller son los autores más reconocidos y citados en este campo (497 y 323 citaciones en artículos científicos respectivamente desde el año 1993) (Proquest, 2004), y ambos hacen referencia a Farquhar como base para sus modelos. Por su parte, Faircloth desarrolla un estudio, que operacionaliza los conceptos de Capital de Marca y realiza una prueba empírica al modelo conceptual adaptado del trabajo de Aaker (1992) y de Keller (1993). La fase final, presenta en forma de conclusiones una comparación de los diversos puntos de vista de cada autor y la teoría de comportamiento del consumidor, para su posterior integración y estimación.

\section{Antecedentes de las Orientaciones de Capital de Marca}

Con base en las afirmaciones de Aaker (1992) se han desarrollado dos orientaciones para analizar y dar respuesta al concepto de Capital de Marca: la financiera y la de comportamiento del consumidor.

La perspectiva financiera está basada en estimar el Capital de Marca para razones netamente contables o para tomar decisiones relacionadas, por ejem- plo, con fusiones o adquisiciones. A partir de la década de los 80 , los montos pagados para la adquisición de marcas reconocidas fueron incrementándose cada vez más, hasta superar el valor de los activos tangibles netos de una compañía (Seetharaman et al. 2001). Como muestra de ello, las estimaciones realizadas por Interbrand, demuestran que el ratio entre el Capital de Marca y la capitalización del mercado es de hasta el $59 \%$ en empresas como Coca Cola, y de $77 \%$ para organizaciones como Nike o Apple (Fernández, 2002).

En esta perspectiva financiera, autores como Farquhar e ljiri (1993); Holbrook (1992); Moran (1994), Simon y Sullivan (1993) y Seetharaman et al. (2001), ofrecen algunas definiciones que hacen referencia a manifestaciones económico-financieras de la marca desde un punto de vista empresarial.

La otra concepción de Capital de Marca viene relacionada con el comportamiento del consumidor. Su principal interés es aumentar la eficacia y la productividad de la actividad de mercadeo en un entorno de aumento de la competitividad, mayores costos de comercialización y una desconcentración de la demanda en muchos mercados (Keller, 1993). Por ello, los departamentos de mercadeo necesitan comprender a mayor profundidad el comportamiento de consumidor para tomar decisiones sobre segmentación y posicionamiento de los productos, y sobre la mezcla de mercadeo (Keller, 1993).

En esta perspectiva, autores como Farquhar (1989), Aaker (1992), Keller (1993), Park y Srinivasan (1994) y Faircloth et al. (2001) desarrollan algunas definiciones donde elementos del compor- 
tamiento del consumidor impactan directamente sobre el valor de la marca.

\section{Teoría del Comportamiento del Consumidor}

El Comportamiento del Consumidor es definido "como una serie de actividades mentales y físicas que llevan a cabo los individuos que resultan en decisiones y acciones para comprar, pagar y usar productos y/o servicios" (Sheth et al, 1999:5). Las organizaciones con una clara orientación al mercado se enfocan primordialmente en entender la dinámica de necesidades y deseos de los consumidores, siendo especialmente el proceso de toma de decisiones de compra el punto clave y focal del estudio del mercadeo y de toda la Teoría del Comportamiento del Consumidor (Engel et al, 1990; Dolan, 1995; Loudon y Della Bitta, 1995; Sheth et al, 1999; Sheth et al, 2003).

Los modelos contemporáneos del Proceso de Toma de Decisiones, ponen en relieve la actividad mental que tiene lugar antes de efectuar la compra, durante ella y después de la misma; por lo que se ven obligados a tomar muchos elementos de otras ciencias del conocimiento, como la psicología y la sociología (Loudon y Della Bitta, 1995). Entre los modelos más conocidos encontramos el Diagrama de Nicosia (1966), quien fue el primero en presentar el proceso como diagrama de flujo. Luego el Modelo de Howard-Sheth (1969) que realiza el primer intento formal en unificar en un mismo esquema todas las alternativas del proceso de compra individual y organizacional; y finalmente el Modelo de Engel-Blackwell-Miniard (1990) que es en la actualidad el modelo más utilizado en la Teoría del Consumidor para explicar el proceso de toma de decisiones (Engel et al, 1990; Loudon y Della Bitta, 1995; Zikmund and d'Amico, 1998; Sheth et al, 1999; Shimp, 2002; Sheth et al, 2003; Boone y Kurtz, 2003).

El Modelo Engel-Blackwell-Miniard (1990), basado en el Modelo de HowardSheth (1969), describe el comportamiento del consumidor como un proceso que se realiza a lo largo del tiempo, y que involucra cinco etapas: 1) Reconocimiento del Necesidad, 2) Búsqueda de Información, 3) Evaluación de Alternativas, 4) Compras y 5) Comportamiento Post-Compra.

El esquema que aparece en la Diagrama 1, agrupa en dos variables diferentes: la entrada y procesamiento de la información, y las variables que influyen en el proceso de toma de decisión. Las flechas del modelo indican el flujo del proceso y las direcciones de influencia ejercidas por cada variable. En la siguiente explicación del modelo se caracterizan la función de cada variable.

Los autores argumentan que el proceso se inicia cuando un individuo $R e$ conoce una Necesidad partiendo de tres influencias posibles: su memoria, factores ambientales y su personalidad. La activación ocurre cuando la persona es capaz de advertir su estado actual y el estado deseado. Una vez activada esta fase, el consumidor inicia la Búsqueda de Información, y para ello emplea dos etapas de acceso, la primera es la Búsqueda Interna, donde va hasta su memoria para recopilar lo que conoce sobre las alternativas para solucionar su necesidad o deseo. Si no esta satisfecho con ese conocimiento actual, realiza una Búsqueda Externa. Como esta fase de búsqueda 


\section{Diagrama 1 \\ Modelo de Comportamiento del Consumidor}

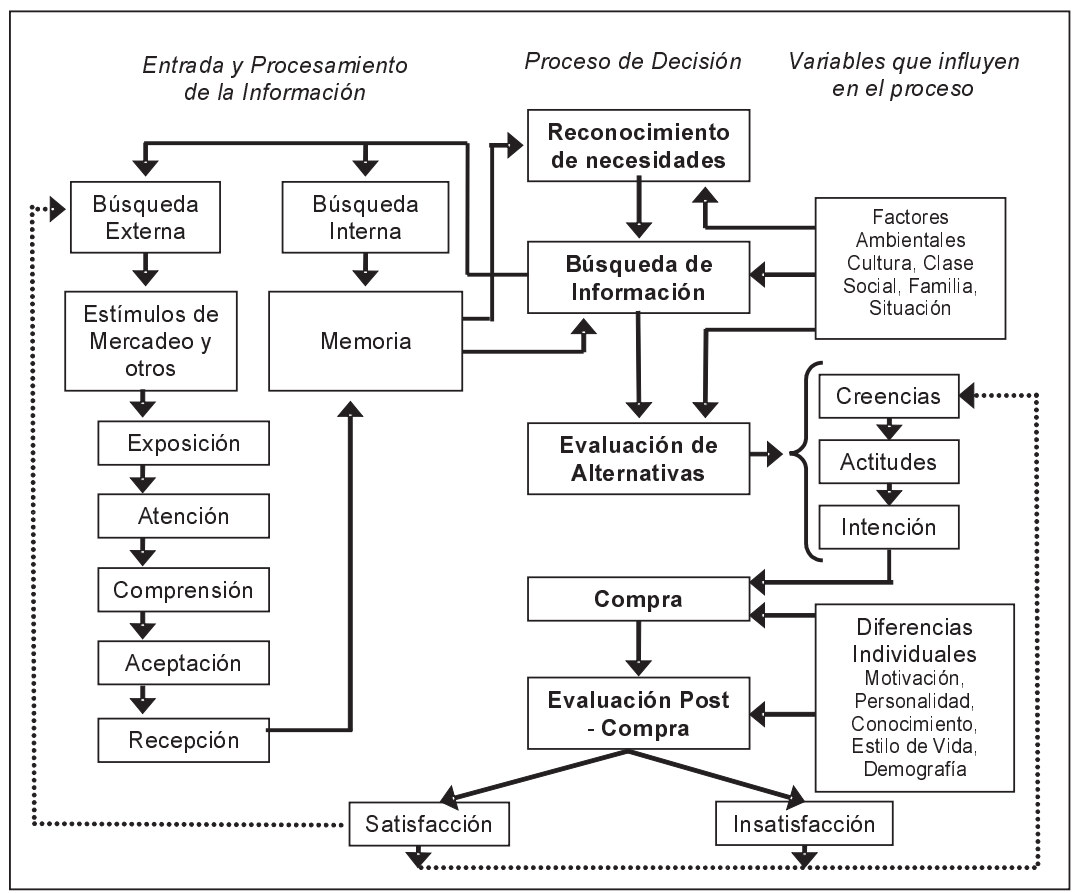

Fuente: Engel, Blackwell y Miniard (1990).

forma parte de la variable entrada y procesamiento de la información, se completa cuando el consumidor decide racionalmente Exponerse y Prestar Atención tan sólo a una serie de estímulos o mensajes que pueden proveerle información. Una vez realizada la misma, el estímulo debe permitir la comprensión del mensaje enviado para que el individuo pueda Aceptar el ofrecimiento del producto o servicio, previo análisis y comparación con otras alternativas, hasta generar el nivel de Recepción óptimo positivo para que la información sea almacenada en la memoria y sirva de insumo para tomar decisiones o sencillamente el óptimo negativo para que la información sea desechada previo análisis y comparación con otras alternativas (Engel et al, 1990).

Al obtener la información necesaria se activa la siguiente fase que es la Evaluación de Alternativas donde se juzgan las diversas opciones existentes con base en las Creencias y Actitudes del individuo. Este proceso genera una Intención de Compra para la marca que recibió la valoración más favorable. A menos que suceda alguna situación externa distinta, la intención debe finalizar en la Compra. Es en este momento que el consumidor empieza a utilizar el producto y Evalúa el rendimiento contra sus expectativas. Un 
posible resultado es la Satisfacción, pero si el producto o servicio no cumplen con las expectativas se generará la Insatisfacción. Este último resultado puede llevar a cabo una nueva Búsqueda Externa, e independientemente, ambos resultados afectan directamente las Creencias del consumidor (Engel et al, 1990).

Finalmente, Engel et al (1990) sostienen que este proceso es continuo y que no culmina con la primera compra, sino que evoluciona con el tiempo. Es en este evolucionar que entra el concepto de la Marca en el Proceso de Toma de Decisión de Compra, ya que la Satisfacción producida en la fase de la Evaluación Post-Compra genera compras repetidas creando la Lealtad de Marca, definida comúnmente como "una serie de compras repetidas de forma consistente impulsada por una disposición psicológica interna del individuo hacia la marca" (Sheth et al, 1999:700). La lealtad de Marca puede medirse, a través de cinco etapas: 1 ) rechazo, 2) no reconocimiento, 3) reconocimiento, 4) la preferencia al ser comparada con otras competidoras; y 5) la insistencia o disposición extrema de consumo de la marca (McCarthy y Perreault, 2001; Boone y Kurtz, 2003).

Generar esta lealtad permite a las marcas influir en el proceso de decisión de compra. En la fase de Reconocimiento de la Necesidad se constituye en una referencia que, archivada en la memoria del consumidor, permite contrastar de inmediato el estado actual con un estado deseado, que previamente ya ha sido probado y verificado por anteriores compras. Esta memoria impulsa al consumidor a una Búsqueda de Información (segunda fase) nula o de baja intensidad, lo que impide a marcas competidoras acceder a través de los estímulos de mercadeo a la fase de Evaluación de Alternativas, ya que el individuo motivado por una experiencia satisfactoria previa no analiza o compara productos, sino que basado en sus Creencias, tiene una Actitud Positiva hacia la Compra de la marca conocida. Finalmente su Evaluación Post-Compra hará que este proceso se profundice al mantener el nivel de satisfacción esperado (Loudon y Della Bitta, 1995; Zikmund and d'Amico, 1998; Sheth et al, 1999; Shimp, 2002; Sheth et al, 2003; Boone y Kurtz, 2003).

La Teoría del Comportamiento del Consumidor hace una clara distinción entre los conceptos de Lealtad y Capital de Marca. Lealtad de Marca es una serie de compras repetidas de forma consistente acompañadas por una actitud favorable hacia la marca. Capital de Marca, por su parte es un concepto mental que le adjudica un nivel de superioridad a la marca. Existen dos grandes diferencias entre ambos conceptos: 1) el Capital de Marca no incluye la dimensión de "compra" y 2) El componente de La Lealtad, actitud favorable, es un concepto mental lo que pudiese ser confundido con el Capital de Marca, pero por ser una "actitud" por definición constituye una evaluación general de la marca y una predisposición a su compra. En contraste, el Capital de Marca está basado más en asociaciones específicas que el consumidor realiza sobre la marca y el valor que dichas asociaciones representan para sí mismo (Sheth et al, 1999; Sheth et al, 2003). 


\section{Propuesta de Peter $\mathrm{H}$. Farquhar (1989)}

El modelo presentado por Farquhar (1989) fue el primero en tratar de explicar y conceptuar las diversas formas para construir y medir el Capital de Marca. Farquhar (1989) parte de la definición de Capital de Marca como el valor agregado con el cual la marca dota al producto. A partir de allí, realiza un análisis de los tres actores que pueden recibir beneficios de dicho valor agregado: La Firma, el Canal de Comercialización y el Consumidor.

El esquema del Modelo de Farquhar (1989) que aparece en la Diagrama 2, agrupa las tres variables antes mencionadas y en la cual observamos que el autor centra su análisis en el consumidor, al desarrollar extensamente dicha variable. Las flechas del modelo indi- can la operacionalización y la influencia de cada variable. En la siguiente explicación del modelo se caracterizan la función de cada variable.

Al analizar las variables Firma y Canal de Comercialización Farquhar (1989) explora conceptos relacionados con la perspectiva financiera del Capital de Marca, al afirmar que la forma idónea de medir dicho concepto es a través del flujo de liquidez incremental que se obtiene al asociar la marca al producto, coincidiendo con Holbrook (1992) y Simon y Sullivan (1993); además de ser la base para un posterior artículo científico (Farquhar e ljiri, 1993).

Farquhar (1989) considera que el Capital de Marca genera ventajas competitivas para la firma, directamente como en su capacidad para negociar con el Canal de Comercialización, como: generar la suficiente fortaleza para superar

\section{Diagrama 2}

\section{Modelo de Capital de Marca de Farquhar}

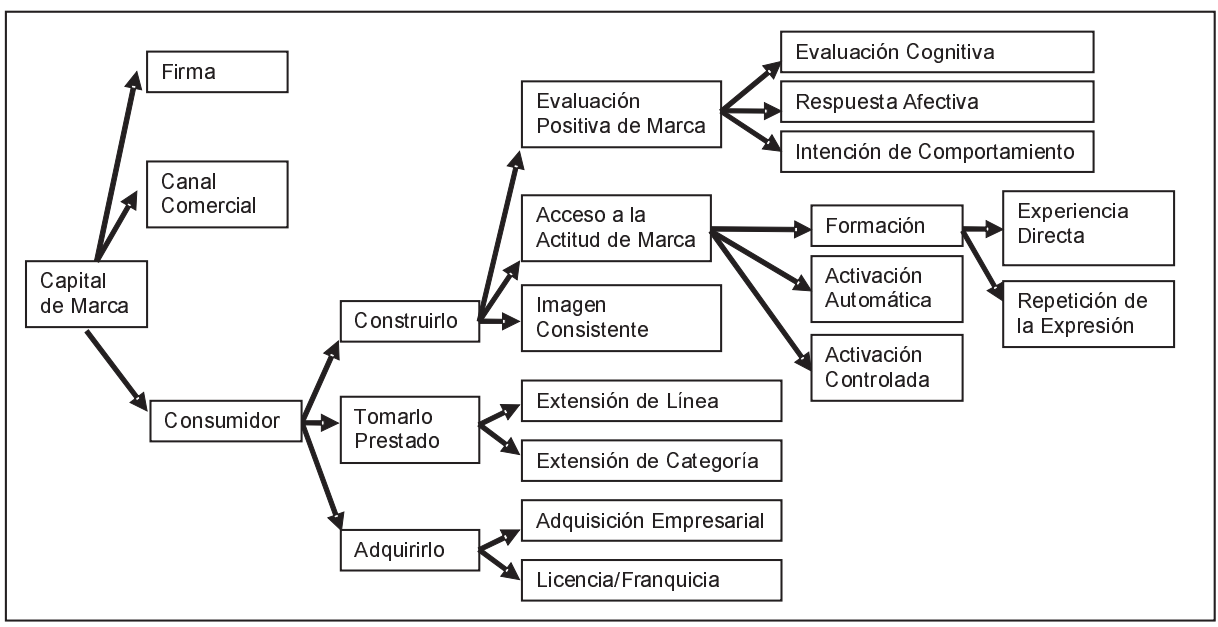

Fuente: Farquhar (1989). 
una crisis (interna o externa) y/o cambios en el gusto del consumidor; la plataforma para el lanzamiento de nuevos productos, franquicias o licencias con una aceptación más rápida y fácil de los canales de distribución, preferencias del canal hacia marcas establecidas por sobre marcas privadas y el establecimiento de barreras de entrada para nuevos productos. Este último aspecto, coincide con los conceptos de Lealtad de Marca expresados por la Teoría del Comportamiento del Consumidor previamente (Sheth et al, 1999; Sheth et al, 2003).

La variable Consumidor es elaborada con mayor amplitud por el autor, al integrar en este punto las tres formas de generar el Capital de Marca: Construirlo, Tomarlo Prestado o Adquirirlo, ya que argumenta que el Capital de Marca se refleja en una mayor actitud positiva del consumidor a utilizar el o los productos de la marca reconocida. Farquhar (1989) define actitud como la asociación entre un objeto (la marca) y la evaluación que de él ha sido almacenada en la memoria del individuo. Aquí encontramos la primera diferencia entre Farquhar y la Teoría de Comportamiento del Consumidor, ya que el autor maneja indistintamente las categorías de actitud, asociación y evaluación; que son claramente establecidas por Loudon y Della Bitta (1995), Sheth et al (1999 y 2003); acercándose a una definición de Lealtad de Marca.

En la operacionalización de la dimensión Construir aparecen tres elementos: 1) Evaluación positiva de la marca; 2) Actitud de marca accesible e 3) Imagen de marca constante. Para obtener una Evaluación Positiva en la mente del consumidor, se debe entregar al consumidor un producto con un desempeño superior a sus expectativas (Farquhar, 1989). EI autor coincide con los conceptos de la Teoría de Comportamiento de Consumidor que asume que la calidad percibida en los productos contribuye a generar un mayor nivel de recordación y percepción en los consumidores (Boone y Kurtz, 2004).

Basado en este desempeño el consumidor podrá realizar su evaluación de tres maneras: evaluación cognoscitiva (inferencias hechas con base a lo que el consumidor "cree" sobre el producto y/o la marca), respuestas afectivas (emociones o sensaciones hacia la marca) y las intenciones conductuales (desarrollo de hábitos de compra). Esta afirmación está validada por el concepto de "Jerarquía de los Efectos Comunicacionales" (Zikmund and D'Amico, 1998; Shimp, 2002; Sheth et al, 2003; Boone y Kurtz, 2003), que organiza sistemáticamente en siete fases el proceso de compra: Ignorancia, reconocimiento, conocimiento, conexión, preferencia, convicción y compra.

Farquhar (1989) comenta que si bien las evaluaciones positivas son fundamentales para construir una marca sólida, no son suficientes sin la actitud apropiada. Una Actitud Accesible es el tiempo que le toma a un consumidor obtener una evaluación almacenada en su memoria. Para ello existen dos modos: Activación Automática, ocurre espontáneamente cuando el individuo es expuesto al producto o marca; y Activación Controlada, donde se necesita la atención del individuo para traer al presente la evaluación (Farquhar, 1989). En el modelo esta activación es "la pieza clave" ya que el objetivo de una marca es fomentar actitudes 
accesibles para afectar el comportamiento del consumidor. Para ello, existen dos estrategias: la Experiencia Directa del Comportamiento (prueba del producto) y la Repetición de la Expresión Actitudinal, que son los esfuerzos coordinados para realzar los puntos positivos de la experiencia directa (e.g. reforzamiento publicitario) (Farquhar, 1989).

El último punto de la dimensión Construcción es mantener una Imagen Constante, ya que es la base para manejar la relación entre el consumidor y la marca. Según Farquhar (1989) esta relación se establece entre la personalidad de la marca y la personalidad del consumidor en cada compra del producto.

En el desarrollo de los conceptos de Actitud Accesible e Imagen Constante, se observan claras similitudes con el proceso de decisión de compra de la Teoría del Consumidor, específicamente en las fases de Búsqueda de Información y Evaluación de Alternativas; lo que refleja que el modelo de Farquhar establece directrices definidas para establecer el Capital de Marca como un constructo de índole mental y psicológica.

Las otras dos dimensiones desarrolladas por Farquhar (1989) son Tomar Prestado y Adquirir el Capital de Marca. Ambos desarrollos enlazan la orientación financiera del Capital de Marca con la orientación del Consumidor, al afirmar que los individuos estarían más dispuestos a aceptar un nuevo producto bajo la denominación de una marca conocida y establecida, que uno con un nombre completamente nuevo (McCarthy y $\mathrm{Pe}$ rreault, 2001). Con base a esto, decisiones sobre extensiones de línea (colocar una marca reconocida a un producto ya existente) o de categoría (colocar el nombre de marca reconocida a una nueva categoría de productos), así como la Adquisición de Empresas, Licencias o Franquicias para marcas de terceros de tienen todo un sentido económico para la organización y para el cliente (Farquhar, 1989).

\section{Propuesta de David Aaker (1992)}

Aaker (1992) presenta una importantísima evolución con respecto al modelo de Farquhar (1989), ya que presenta al Capital de Marca como un conjunto de activos íntimamente ligadas al nombre y al símbolo (logotipo) de la marca, que pueden (y deben) ser manejados por la gerencia de mercadeo para generar valor. La base de la propuesta de Aaker es que cada activo crea valor para el cliente y para la organización de distintas maneras. Por ello, la forma de ser eficientes es entender como se crea el valor en cada etapa y con cada componente, para de esa forma tomar decisiones coherentes (Aaker, 1992).

El esquema que aparece en la Diagrama 3, presenta los cinco activos de la marca: lealtad, conciencia, calidad percibida, asociaciones y otros activos; así como dos mecanismos para crear valor, para el cliente, y la organización. Las flechas del modelo indican el flujo del proceso y la influencia de cada variable. En la siguiente explicación del modelo se caracterizan la función de cada una de ellas.

Aaker (1992) es el primer autor en incorporar el concepto de Lealtad a su modelo de Capital de Marca, mantenien- 


\section{Diagrama 3 \\ Modelo de Capital de Marca de David Aaker}

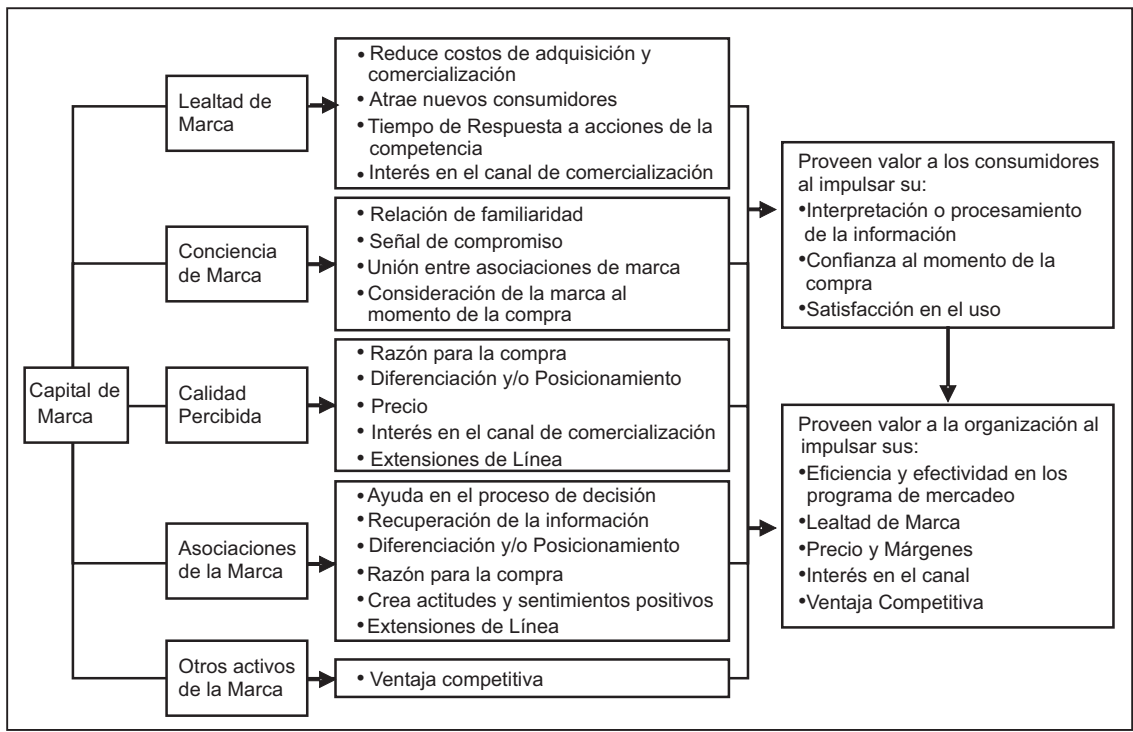

Fuente: Aaker (1992).

do la misma definición de la Teoría de Comportamiento del Consumidor analizada previamente. Aaker (1992) afirma que este activo genera valor al reducir directamente los costos de adquisición y comercialización, ya que retener a clientes actuales es menos costoso que adquirir nuevos consumidores. A mayor fidelidad de marca, menor vulnerabilidad de los clientes a los estímulos de mercadeo de la competencia, ya que tienen poca o ninguna motivación a evaluar nuevas alternativas. Además, clientes leales se convierten en portavoces de la marca. En estos puntos Aaker (1992) coincide con Sheth et al (2003) y Boone y Kurtz (2003).
El activo Conciencia es la capacidad de un cliente para reconocer o recordar que una marca es miembro de una categoría de producto. En este desarroIlo, Aaker (1992) sintetiza el esquema de los cinco niveles de Lealtad de Marca en sus componentes racionales, para afirmar que desde su nivel más superficial (reconocer), este activo provee al consumidor un sentido de familiaridad y de compromiso para con la marca, ya que el simple hecho de reconocerla afecta dramáticamente sus evaluaciones. Cuando la Conciencia se encuentra a un nivel muy elevado (conocimiento), obvia la fase del proceso de decisión de compra de Evaluación de Alternativas, para afec- 
tar directamente las elecciones y la toma de decisión del consumidor.

En el activo Calidad Percibida, Aaker mantiene la concepción de la Teoría de Comportamiento de Consumidor que plantea que la calidad percibida en los productos o servicios contribuye a generar un mayor nivel de recordación y percepción en los consumidores (Boone y Kurtz, 2004). A este concepto, Aaker (1992) añade que la calidad percibida genera una percepción de superioridad (o inferioridad) al producto o servicio con respecto a un sustituto. Esto genera valor a todos los actores involucrados en su comercialización: el cliente encuentra una razón para comprar el producto, el canal de distribución se siente atraído a comercializarlo y la firma encuentra una base para generar extensiones de línea y colocar un precio más elevado.

En su modelo, Aaker (1992) incorpora por primera vez el concepto de Asociaciones en el esquema de Capital de Marca, manteniendo una clara diferenciación entre este concepto y el de Lealtad, al igual que la Teoría del Comportamiento de Consumidor (Sheth et al, 1999; Sheth et al, 2003). Aaker (1992) define las Asociaciones como una serie de emociones, imágenes, sonidos, etc.; vinculados al recuerdo de la marca, que ayudan a los clientes a recuperar información archivada en su mente para tomar decisiones y le proveen una razón para adquirir el producto, generándole sentimientos positivos (fase de evaluación de alternativas del proceso de decisión de compra, Engel et al, 1990).

Una característica fundamental de las Asociaciones es que poseen un nivel de fuerza o intensidad. El vínculo entre la marca (producto) y la asociación será mas sólida si se basa en experiencias directas, en exposiciones a esfuerzos de comunicación o cuando se está apoyado por una secuencia de otros vínculos (Aaker, 1992).

Los Otros Activos del modelo de Aaker (1992) están constituidos por aquellos elementos tangibles asociados a la marca, al producto en sí mismo o a la organización que puedan afectar positiva o negativamente la creación de valor. Entre estos activos se encuentran las patentes, la propiedad intelectual y la experticia organizacional (Aaker, 1992).

La actuación al unísono de los activos de la marca genera mecanismos para la creación de valor para los clientes y los accionistas de la organización. A nivel de consumidor se generan tres: 1) los activos ayudan a interpretar, procesar, almacenar y recuperar información sobre los productos y las marcas en el mercado; 2) los activos de la marca pueden afectar la seguridad de la toma de decisión de un consumidor, ya que éstos se sienten más seguros al usar marcas conocidas o que les generen pensamientos positivos; $y$ 3) cuando el cliente utiliza el producto se refuerza el nivel de satisfacción experimentado a través de la percepción de calidad y las asociaciones de marca (Aaker, 1992).

A nivel de la organización, Aaker (1992) detalla seis mecanismos: 1) el Capital de Marca aumenta la eficacia y la eficiencia de los programas de mercadeo; 2) el conjunto de calidad percibida, conciencia y asociaciones de marca; desarrollan y consolidan la lealtad de los consumidores, incrementando su satisfacción y dándoles razones para comprar el 
producto; 3) permite fijar precios mayores, a la vez que reduce la dependencia de las promociones para aumentar las ventas; generando márgenes de ganancia más altos; 4) establece la plataforma para extensiones de línea; 5) Traspaso de las asociaciones de marca a la firma, otorgando una mejor percepción al canal de comercialización, para negociar con la organización dueña de una marca reconocida; y 5) los activos proporcionan una ventaja significativa organizacional al establecer barreras para la retención de los clientes (Aaker, 1992).

\section{Propuesta de Kevin Lane Keller (1993)}

El modelo Keller (1993) toma los postulados de Farquhar (1989), Aaker (1992) y la Teoría del Comportamiento para introducir un nuevo concepto: Capital de Marca Basado en el Consumidor (CMBC), que es definido como el efecto diferencial del conocimiento de marca por parte del consumidor como resultado de sus esfuerzos de comercialización. La base de la propuesta de Keller (1993) es que una marca posee CMBC positivo (negativo) si el consumidor reacciona muy (poco) favorablemente a esfuerzos de mercadeo orientados a la marca, de lo que lo haría si es expuesto al mismo estímulo atribuido a una marca ficticia o un producto con nombre genérico (Keller, 1993).

El esquema que aparece en la Diagrama 4, presenta la descomposición de la Variable Conocimiento de Marca, en sus dos componentes básicos: Conciencia e Imagen. Las flechas del modelo indican el flujo del proceso y la influencia de

\section{Diagrama 4 \\ Capital de Marca Basado en el Consumidor (CMBC)}

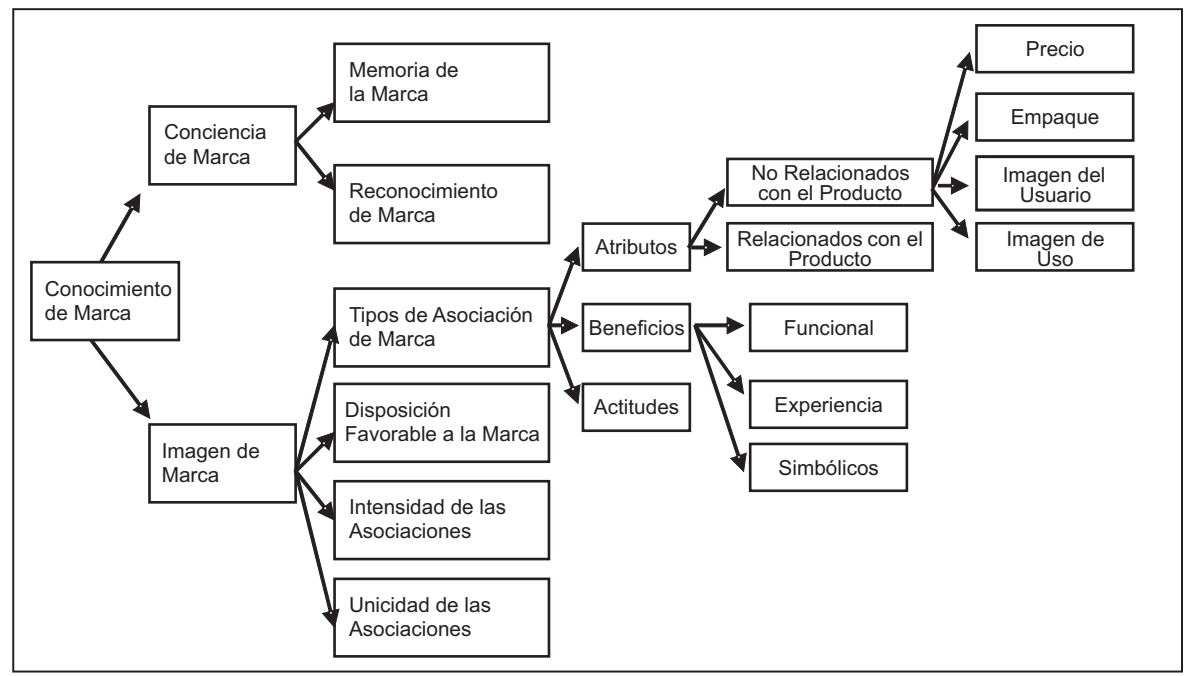

Fuente: Keller (1993). 
cada variable. En la siguiente explicación del modelo se caracterizan la función de cada una de ellas.

Keller (1993) realiza una profunda conceptualización de la variable Conocimiento de Marca, ya que el basamento es que el CMBC existe sólo cuando el consumidor mantiene una relación cercana con la marca, a la vez que realiza asociaciones favorables, fuertes, y diferenciadas de ella en su memoria. Es el Conocimiento la variable que permite abordar los mecanismos generados en la mente del consumidor cuando piensa en la marca, y es definido en dos dimensiones: Conciencia e Imagen de Marca.

La primera de las dimensiones, Conciencia de Marca, es definida por Keller (1993) como la capacidad de los consumidores para identificar la marca bajo diversas condiciones y está constituida por el reconocimiento (capacidad de identificar los elementos gráficos de la marca en presencia física del producto) y memoria de la marca (capacidad de identificar los mismos elementos sin la presencia física del producto o la marca). Ambos conceptos, se relacionan con la segunda fase del comportamiento de compra, Búsqueda de Información, que presenta dos componentes: la búsqueda externa que incluye aspectos y estímulos de mercadeo (logo, merchandising, ideales para lograr el reconocimiento) y la búsqueda interna que orienta a la recordación de productos, marcas y experiencias directamente de la memoria (Sheth et al, 1999; Sheth et al, 2003).

Keller (1993) define la segunda dimensión, Imagen de Marca, como las percepciones que sobre la marca son reflejadas a través de las distintas asocia- ciones de marca. Este apartado se desarrolla en dos grandes áreas: por un lado se operacionaliza los tipos de asociación posibles: Atributos, Beneficios y Actitudes. Por el otro, se explican las tres características de las mismas: Disposición favorable, Intensidad y Unicidad de la asociación.

Keller (1993) divide las asociaciones por Atributos en dos: 1) Atributos No Relacionados con el Producto, donde incluye aspectos como precio, empaque, imagen del usuario y de uso; y 2) Atributos Relacionados con el Producto, donde se incluyen aspectos como tamaño, calidad, entre otros. Las asociaciones por Beneficios se dividen en Beneficios Funcionales (desempeño), Beneficios por Experiencia (evaluación personal) y Beneficios Simbólicos (conceptos individuales y/o grupales, como lujo, status, pertenencia). Finalmente, las asociaciones por Actitudes son las conexiones entre la personalidad del consumidor y la personalidad de la marca. En el caso de ésta última asociación, el autor maneja el concepto similar al utilizado en la Teoría del Comportamiento del Consumidor, definiendo Actitud como "predisposiciones aprendidas que impulsan a responder de forma consistente de forma positiva o negativa hacia un producto" (Sheth et al, 1999: 388).

Todas los tipos de Asociaciones planteados por Keller (1993) se encuentran suscritos en las dos variables del proceso de decisión de compra del consumidor: 1) Entrada y Procesamiento de la Información (Asociación Relacionada y No Relacionada con el Producto y por Beneficios Funcionales), y 2) Variables que influyen en el proceso (Asociación por Acti- 
tudes, Beneficios por Experiencia y Beneficios Simbólicos.

Una vez definido el set de Asociaciones de una marca, Keller (1993) clasificó sus dimensiones como: Disposición Favorable (positiva o negativa), Intensidad (alta, baja, inexistente) y Unicidad (asociación única, no compartida con otras marcas). De estas tres dimensiones, tan sólo la última corresponde a un desarrollo propio de las asociaciones, mientras que Disposición Favorable e Intensidad son tomadas por Keller de las características de las Actitudes.

Una vez conceptualizado el modelo, se hace referencia a los beneficios organizacionales del CMBC, que pueden impulsar el fortalecimiento de los márgenes de ganancia, costos más bajos y mayores beneficios. EI CMBC contribuye también a aumentar la probabilidad de compras repetidas, generando lealtad del consumidor, impulsando la disposición del cliente a usar diversos puntos de ventas para la obtención del producto, aumentando la efectividad de la promoción, disminuyendo la vulnerabilidad de la marca ante acciones de la competencia y creando las bases para desarrollar franquicias, acuerdos de licencias y estrategias de extensión de línea (Keller, 1993).

Finalmente, Keller (1993) desarrolla líneas de acción para Construir, Evaluar y Gerenciar el CMBC. Construir una relación entre el consumidor y la marca que contenga disposiciones favorables positivas, con alta intensidad y asociaciones únicas, se origina con una cuidadosa selección inicial de la identidad de marca a ser integrada al plan de mercadeo. Para la Evaluación existen dos aproximacio- nes: 1) La Indirecta, orientada a medir el conocimiento de la marca para determinar las fuentes potenciales de CMBC; y 2) La Directa que mide los efectos del conocimiento de la marca en la respuesta del consumidor motivado por los esfuerzos de mercadeo (Keller, 1993).

Finalmente para Gerenciar el CMBC se desarrollan seis pautas: 1) construir una visión a largo plazo de la gerencia de la marca; 2) priorizar los deseos del consumidor dentro de los beneficios centrales de la marca; 3) considerar un amplio rango de alternativas promocionales; 4) desarrollar un esquema de control para mantener cohesionados y coordinados los esfuerzos de mercadeo; 5) realizar periódicamente estudios y mediciones; y 6) finalmente evaluar el potencial para futuras extensiones de línea o marca (Keller, 1993).

\section{Propuesta de Faircloth, J.L.; Capella, L.M. y Alford, B.L. (2001)}

El principal objetivo del trabajo presentado por Faircloth et al (2001) fue demostrar que las continuas críticas realizadas al concepto de Capital de Marca referidas a su poca (o ninguna) relevancia para el mundo gerencial y empresarial estaban infundadas. Para ello, los autores operacionalizaron los conceptos adaptados de los trabajos de Aaker (1992) y Keller (1993), para realizar una prueba empírica, donde manipularon, utilizando un modelo de ecuación estructural, diferentes asociaciones de marca con especial énfasis en el efecto de la actitud y la imagen sobre el Capital de Marca. 
Los hallazgos de Faircloth et al (2001) se ubican en dos dimensiones, al categorizar la Actitud y la Imagen tanto como indicadores y como antecedentes del Capital de Marca. La definición de Indicadores es desarrollada al encontrar suficiente evidencia de que una Imagen de Marca positiva acrecienta la intención de compra en los consumidores, así como una mayor disposición a pagar precios superiores. Este rol de Indicador tiene coherencia con la Teoría del Comportamiento del Consumidor especialmente en las áreas de Lealtad de Marca, Imagen Social (percepción de estima referida al grupo que utiliza la marca) e Identificación (nivel en el cual los consumidores se sienten identificados con la marca) (Sheth et al., 1999).

El rol de Antecedentes de la Imagen y la Actitud es construido por Faircloth et al (2001) al encontrar suficiente evidencia de que ambos conceptos pueden ser objeto de manipulación por parte de la organización a través de las herramientas de la mezcla de mercadeo. Este hallazgo validó la hipótesis de la investigación, al demostrar que el Capital de Marca no sólo es relevante en el mundo empresarial, sino que una parte de sus indicadores son responsabilidad directa de los gerentes de mercadeo.

Al obtener resultados que proporcionaron una confirmación parcial de la teoría presentada por Aaker (1992) y Keller (1993), y validar su hipótesis, Faircloth et al. (2001) desarrolla una propuesta de cuatro para crear Capital de Marca:

1) Gerenciar los aspectos que lo crean, 2) Capacidad de manipulación de las asociaciones, 3) Aplicación de múltiples tácticas de comercialización e 4) Inver- sión en imagen para empresas de e-commerce.

Primeramente Faircloth et al. (2001) expone que los gerentes de mercadeo únicamente deben Gerenciar los Aspectos con los que se construye el Capital de Marca, ya que si la sola construcción de una imagen afecta este capital, las firmas deberían crear imágenes de marca que hayan demostrado tener efectos positivos en el mismo. En segundo lugar, cuando los gerentes entienden que las Asociaciones pueden ser Manipuladas para crear la imagen deseada a través de acciones de comunicación específicas por asociación y segmento de consumidores, el Capital de Marca se ve reforzado.

En tercer lugar Faircloth et al. (2001) explica que el Capital de Marca es una medida de la eficiencia en la Aplicación de Tácticas de Comercialización. El autor sugiere realizar estudios de sensibilidad por cada táctica manipulando la actitud y la imagen de la marca, y así analizar qué combinación genera la asociación más adecuada (alta correlación) para el Capital de Marca deseado. Finalmente, los autores recomiendan un cuidadoso análisis de Inversión de Imagen para las empresas del E-commerce, ya que a pesar de las características de la Internet, la Marca sigue siendo el factor fundamental para la toma de decisiones de compra por parte del consumidor.

\section{Comparación de Modelos Teóricos de Autores}

Al revisar los trabajos individuales de los cuatro autores, podemos agrupar sus aportes, recomendaciones y méto- 
dos en tres grandes áreas: 1) las Fuentes que generan el Capital de Marca, 2) los Beneficios (y los beneficiarios) de la existencia de Capital de Marca y 3) los Métodos para su Construcción.

Este esquema de análisis permitirá desarrollar de forma particularizada el contraste y la integración de la teoría alrededor del concepto de Capital de Marca, haciendo la salvedad del estudio de Faircloth et al. (2001) ya que por ser una comprobación empírica de los modelos de Aaker y Keller, este autor no propone un set diferente de fuentes, ni beneficios. Por el contrario se apoya en los demás autores para proponer formas de gerenciar dichas fuentes de generación de Capital de Marca.

\subsection{Comparación de las Fuentes que generan el Capital de Marca}

De los tres autores, el modelo de Aaker (1992) es el que presenta mayor profundidad a la hora de exponer las Fuentes que generan el Capital de Marca, ya que si cada activo (lealtad, conciencia, calidad percibida, asociaciones y otros) crea valor para el cliente y la firma de distintas maneras; cada uno de ellos es en sí mismo una fuente de generadora de Capital de Marca. Bajo esta óptica el trabajo de Aaker (1992) desarrolla aspectos cognitivos (conciencia y calidad percibida), afectivos (asociaciones), de comportamiento (lealtad de marca) y organizacionales (otros activos); creando un sólido marco de referencia para trazar estrategias.

Por su parte, en su modelo Farquhar (1989) desarrolla tres fuentes de Capital de Marca (evaluación positiva de la marca, acceso a la actitud e Imagen consistente), siendo la primera la de mayor importancia para obtenerlo, ya que a pesar de diferenciar esta evaluación en tres tipos (afectiva, cognitiva y conductual), el autor comenta que la base fundamental para ello es que la organización desarrolle un producto con el mayor nivel de calidad posible y con un desempeño superior al de la competencia.

Las fuentes de Capital de Marca desarrolladas por Keller (1993) en el modelo CMBC, están concentradas en la operacionalización de la dimensión Asociaciones. Estas Asociaciones son la clave del desarrollo de Keller, siendo las fuentes que la generan: los atributos (relacionados o no) del producto y las actitudes del consumidor.

Al hacer el contraste de aportes encontramos importantes coincidencias entre los postulados de los autores, observándose una mayor vinculación individual de Aaker con Farquhar y Keller, por presentar una mayor profundidad en el tratamiento de Las fuentes de Capital de Marca. La similitud más notable que se encuentra es que tanto las fuentes expuestas por Farquhar como por Keller, están contempladas en el modelo de Aaker siendo desarrolladas a mayor profundidad debido a una mayor integración de las mismas.

Específicamente, Farquhar (1989) y Aaker (1992) coinciden en tres aspectos: 1) desarrollan las fuentes bajo aspectos afectivos, cognitivos y conductuales; 2) presentan conceptos de Identidad de Marca propios de la Teoría de Comportamiento de Consumidor, al asumir por igual que la calidad percibida en los productos contribuye a generar un mayor ni- 
vel de recordación y percepción en los consumidores (Boone y Kurtz, 2004) y 3) exploran el área común de ActitudLealtad. Si bien Aaker (1992) no introduce este concepto en su modelo, considera la Lealtad como una actitud de muy alta intensidad hacia algo o alguien; lo que concuerda con la definición de Lealtad de marca de la Teoría de Comportamiento de Consumidor (Sheth et al., 1999:709).

La diferencia fundamental entre ambos autores radica en que Aaker (1992) no desarrolla en su modelo orientaciones para construir dicha lealtad, contrariamente a lo hecho por Farquhar (1989) que detalla como se forman las actitudes y los métodos para poner en funcionamiento dicha actitud.

Por su parte Keller (1993) y Aaker (1992) coinciden en desarrollar el concepto de Conciencia de Marca y las Asociaciones como un componente de la Imagen de Marca. Sobre ambos puntos, Keller (1993) realiza un aporte posterior a lo expuesto por Aaker. En el concepto de conciencia de marca añade una construcción teórica más detallada al diferenciar los conceptos de reconocimiento y la memoria de la marca, presentando definiciones similares a las de Farquhar (1989), pero ampliándolas con mayor rigurosidad. En el concepto de Asociaciones, Keller (1993) profundiza y determinar qué aspectos (atributos, beneficios y/o actitudes) de la marca generan dichas asociaciones. Esta similitud entre autores ha definido la orientación del Capital de Marca, como un concepto más basado en asociaciones específicas de la marca y a consideraciones de lo que estos valores representan (Sheth et al., 1999).

\subsection{Comparación de Beneficios que generan el Capital de Marca}

Al analizar los Beneficios que generan el Capital de Marca encontramos una marcada coincidencia entre Farquhar, Aaker y Keller. Al definir los beneficios desde el punto de vista organizacional, encontramos: menores costos de adquisición, mayores niveles de retención de clientes debido a mayores niveles de lealtad de marca; así como mayor eficiencia y efectividad en los esfuerzos de mercadeo, una mayor probabilidad de que el consumidor esté dispuesto a aceptar mayores precios por los productos, lo que genera a su vez un aumento de los ingresos y las utilidades; y finalmente una base sólida para extensiones de línea. Estos beneficios poseen una orientación más financiera, que coinciden con apreciaciones de autores como Holbrook (1992); Moran (1994), Simon y Sullivan (1993) y Seetharaman et al. (2001).

En paralelo a estas notorias coincidencias, Aaker (1992) es el único de los autores en conceptualizar los beneficios percibidos por el cliente generados por el Capital de Marca. Los beneficios: nivel de satisfacción, seguridad en la toma de decisiones y pensamientos positivos; son el punto central que permite, por un lado medir la eficiencia de los esfuerzos por generar Capital de Marca, y por el otro, la base para evaluar y predecir los cambios del comportamiento del consumidor y como se refleja ello en las finanzas de la organización. 


\subsection{Comparación de los Métodos para Construir el Capital de Marca}

Al analizar la teoría sobre los Métodos para Construir el Capital de Marca, encontramos una diferencia fundamental con respecto a los otros dos puntos tratados en esta parte. Por un lado se debe a Faircloth (2001) quien desarrolla un plan de acción en este sentido. Por el otro, se excluye a Aaker (1992) quien no desarrolla una metodología para construir capital de marca.

Farquhar (1989) desarrolla tres etapas para construir Capital de Marca: 1) Introducción, 2) Elaboración y 3) Consolidación. El autor deja entrever que las evaluaciones del consumidor (cognitiva, afectiva e intención de compra) tienen un orden y se inician desde un punto de vista racional, ya que la evaluación cognitiva refleja criterios sobre la calidad del producto. Este desempeño superior genera en el consumidor una Evaluación Positiva hacia la marca, y una vez desarrollada esta fase, se abre el compás tanto para la respuesta afectiva como para la intención de compra. Esta afirmación está validada por el concepto de "Jerarquía de los Efectos Comunicacionales" que organiza sistemática y escalonadamente en siete fases el proceso de compra del consumidor (Zikmund and d'Amico, 1998; Sheth et al, 1999; Shimp, 2002; Sheth et al, 2003; Boone y Kurtz, 2003).

Por su parte, Keller (1993) desarrolla seis guías para gerenciar la construcción del Capital de Marca, desde una óptica completamente organizacional: 1) EI establecimiento de una visión a largo plazo, 2) la decisión de colocar los deseos del cliente como beneficio central de la marca, 3) seleccionar diversas alternativas promocionales para cada asociación de marca, 4) el desarrollo de un esquema de control, 5) el uso de estudios y mediciones de campo; y 6) la evaluación de posibles extensiones de línea basados en el capital de marca desarrollado.

Los métodos desarrollados Faircloth (2001) parten de los resultados de su investigación que demostraron que la Imagen y las Asociaciones de Marca son antecedentes e indicadores de Capital de Marca. Por ello, no postula un proceso completo sino que explora (y especialmente orienta) la capacidad de los gerentes de mercadeo para manipular la Imagen y las Asociaciones, centrando su análisis en las distintas posibilidades para conseguirlo.

Al contrastar los aportes de lo autores encontramos que los tres planteamientos son complementarios, ya que para una eficiente construcción de Capital de Marca las organizaciones deben partir con las seis pautas desarrolladas por Keller (1993) como orientación de planificación a corto, mediano y largo plazo; para poder manejar las tres etapas descritas por Farquhar (1989), asumiendo durante la construcción la responsabilidad que tienen los gerentes de mercadeo para manipular la Imagen y las Asociaciones de Marca a través de las técnicas explicadas por Faircloth (2001).

A la par de lo anterior, se encuentran importantes coincidencias entre los postulados de los autores. Por un lado, Keller (1993) coincide con Farquhar (1989) en definir la calidad como un factor necesario para construir Capital de Marca ya que las Asociaciones son determinadas inicialmente por este. Por su parte, 
Faircloth (2001) y Keller (1993) concuerdan que la Imagen y la Identidad son el primer paso del método de construcción de Capital de Marca y que para ello es necesario: 1) Una cuidadosa elección inicial de la identidad de marca (logotipo, símbolos) para desarrollar Asociaciones de Marca favorables, sólidas y únicas; y 2) la aplicación de estudios para la escogencia de los elementos que hayan demostrado tener efectos positivos en el Capital de Marca.

Faircloth (2001) profundiza estos postulados al exponer la necesidad de generar acciones de comunicación por cada asociación, ya que existe un segmento de consumidores que han desarrollado vínculos y evaluaciones tan específicos para con la marca, que ameritan una comunicación personalizada.

\section{Conclusiones}

El concepto de Capital de Marca no constituye un área de fácil abordaje, ya que todos los desarrollos teóricos de su operacionalización se fundamentan en aspectos del comportamiento del consumidor. Por ello, los autores abordan los componentes desde distintas perspectivas que van desde criterios racionales y cognitivos, hasta aspectos de naturaleza emocional; pasando por construcciones generadas por percepciones y apreciaciones particulares de cada individuo. Este desarrollo teórico, profundizado por los trabajos de Aaker (1991) y Keller (1993), consiguió su validación, luego del estudio empírico desarrollado por Faircloth (2001).

El contraste de los autores permitió observar, que si bien el Modelo planteado por Aaker (1992) presenta una mayor amplitud de fuentes para generar el Capital de Marca, éste se complementa por el modelo CMBC de Keller (1993) al presentar un mayor grado de operacionalización de los componentes similares. Es por ello que el Capital de Marca es un concepto que aún no ha desarrollado una convención ampliamente aceptada, ya que los modelos aquí analizados se complementan entre sí a la hora de descomponer y analizar sus elementos constitutivos.

Al analizar bibliografía del área de mercadeo (principios básicos, mercadeo estratégico y comportamiento del consumidor) se encuentra que el modelo de $\mathrm{CMBC}$ de Keller (1993) es ampliamente usado como referencia para sistematizar el concepto de Capital de Marca (Hoffman et al, 2002), pero sin ser completamente estable, ya que cada autor le añade componentes propios de Aaker (1992) y de su propia autoría (Zikmund and d'Amico, 1998; Sheth et al, 1999; Shimp, 2002; Sheth et al, 2003; Boone y Kurtz, 2003).

Esta constate búsqueda y adaptación de los modelos de Capital de Marca, reflejan su valor organizacional cada vez más, en un ambiente altamente competitivo y de expansión del modelo de globalización comercial. Los conceptos de Marca y su Capital, permiten un mayor control de los esfuerzos de mercadeo. Esta afirmación realizada por Faircloth (2001) introdujo, por primera vez, el concepto de Capital de Marca como un elemento de medición de la eficiencia de la actividad de mercadeo; lo que conlleva a estrictos controles y análisis de inversión, permitiendo materializar la tan ansiada promesa de poder medir financiera y económicamente el impacto del desarrollo de las Marcas. 


\section{Referencias Bibliográficas}

Aaker, David A. (1992), "The value of brand equity". Journal of Business Strategy, Vol. 13, Iss. 4, Jul/Aug. USA. Emerald Group Publishing Limited. Pág. 27-32.

Boone, Louise y Kurtz, David (2003), Contemporary Marketing. USA. South-Western College Pub. 11ma. Edición. 624 Págs.

Delgado, Elena (2004), "Controversia conceptual sobre el capital de marca: Propuesta de un marco teórico de análisis". En Revista Europea de Dirección y Economía de la Empresa. Vol. 13, No. 1. España. Asociación Europea de Dirección y Economía de la Empresa.

Dolan, Robert (1995), La Esencia del Marketing. Vol. 1. Estrategia. Colombia. Editorial Norma. 380 Págs.

Engel, James; Blackwell, Roger y Miniard, Paul (1990), Consumer Behavior USA. Dryden Press. 6ta Edición. 592 Págs.

Faircloth, James; Capella, Louis; Alford, Bruce (2001), "The effect of brand attitude and brand image on brand equity". In Journal of Marketing Theory and Practice. Vol. 9, Iss. 3. USA. Association of Marketing Theory and Practice. Pág. 61-75.

Farquhar, Peter (1989), "Managing brand equity". In Marketing Research. Vol. 1, Iss. 3. USA. American Marketing Association (AMA) Publications. Pág. 24-33.

Farquhar, Peter; Ijiri, Yuji (1993), "A dialogue on momentun accounting for brand management". In International Journal of Research in Marketing, Vol. 10. USA. Elsevier Publishing Ltd. Pág. 77-92.

Fernández, Pablo (2002), "Valuation of Brands and Intellectual Capital". Research
Paper. No. 456. España. IESE Universidad de Navarra.

Hoffman, K. Douglas; Czinkota, Michael R.; Dickson, Peter R.; Dunne, Patrick; Griffin, Abbie; Hutt, Michael D.; Krishnan, Bilaji; Lindgren, John H. Hr.; Lusch, Robert F.; Ronkainen, Ikka A.; Rosenbloom, Bert; Sheth, Jagdish y Shimp, Terence A. (2002), Marketing: Best Practices. USA. SouthWestern College Pub. 2da. Edición. 650 Págs.

Holbrook, Morris (1992), "Product quality, attributes, and brand name as determinants of price: The case of consumer electronics". In Marketing Letters. No. 1. Vol. 3. USA. Kluwer Academic Publishers. Pág. 71-83.

Keller, Kevin (1993), "Conceptualizing, measuring, and managing customer-based brand equity". In Journal of Marketing. Vol. 57, Iss. 1. USA. American Marketing Association (AMA) Publications. Pág. 1-22.

Loudon, David y Della Bitta, Albert (1995), Comportamiento del Consumidor. México. McGraw-Hill Interamericana de México. 4ta. Edición. 834 Págs.

McCarthy, Jerome y Perreault, William (2001), Marketing: Un Enfoque Global. USA. McGraw-Hill Interamericana Editores. 13ra. Edición. 797 Págs.

Moran, Williams T. (1994), "Marketplace measurement of brand equity". The Journal of Brand Management. Vol. 1, N. 5. USA. Henry Stewart Publications. Pág. 272-282.

Park, Chan Su; Srinivasan, V. Seenu (1994), "A survey-based method for measuring and understanding brand equity and its extendibility". In Journal of Marketing Research. Vol. 31. USA. American Marketing Association (AMA) Publications. Pág. 271- 288.

Proquest (2004), http://www.proquest.com. Junio, 2004. 
Seetharaman, Ananth; Mohd Nadzir, Zainal Azlan Bin y Gunalan Seethu (2001), "A conceptual study on brand valuation". In Journal of Product \& Brand Management. Vol. 10, No. 4. USA. Emerald Group Publishing Limited. Pág. 243 - 256.

Sheth, Jagdish; Mittal, Banwari y Newman, Bruce (1999), Customer Behavior: Consumer Behavior and Beyond. USA. Harcourt Brace College Publishers. 799 Págs.

Sheth, Jagdish; Mittal, Banwari y Newman, Bruce (2003), Customer Behavior: A Managerial Perspective. USA. South-Western College Pub. 2da. Edición. 550 Págs.
Shimp, Terence A. (2002), Advertising, Promotion and Supplemental Aspects of Marketing Communication. USA. South-Western College Pub. 6ta. Edición. 704 Págs.

Simon, Carol. J.; Sullivan, Mary W. (1993), "The measurement and determinants of brand equity: a financial approach". Marketing Science. Vol. 12, N.1. USA. Marketing Science Institute Pág. 28-52.

Zikmund, William y D'Amico, Michael (1998), Effective Marketing. USA. SouthWestern College Pub. 5ta. Edición. 498 Págs. 